48 SUPPORTING INTERNAL MEDICINE TRAINEES TO MEET NEW CURRICULUM COMPETENCIES AROUND END OF LIFE CARE: A NOVEL SIMULATION COURSE

Ruth Caulkin, Louise Robinson. Chelsea and Westminster NHS Foundation Trust

10.1136/spcare-2021-PCC.66

Introduction The new Internal Medicine Training (IMT) curriculum defines a specific Capability in Practice (CiP) around managing end of life and applying palliative care skills. We have developed a mixed methods program to meet the necessary curriculum competencies, including a simulation course. The aim of this project was to adapt and design a bespoke simulation course and to pilot and evaluate it with IMT trainees

Methods The training was adapted from an existing course, CHOICE (Communicating Honestly and Openly to Improve Care at End of life), a high-fidelity simulation course, modelling a patient's journey at the end of life. Each trainee participates in a simulated scenario with an actor and receives focused feedback from a multidisciplinary faculty. Two half day courses were piloted with a total of eight trainees. The courses were evaluated using pre and post course questionnaires, assessing confidence in several areas related to end of life care using a 5-point Likert scale.

Results In all domains assessed, the IMT trainees reported an improvement in confidence in identifying a patient reaching the end of their life and communicating this with them and their relatives. Feedback demonstrated that trainees found it a safe place to explore and practice conversations around end of life care. $100 \%$ of participants reported the course as 'very much' likely to have an impact on their approach to end of life care. All participants would recommend the course to a colleague.

Conclusion The pilot course was highly valued by IMT trainees to support them to meet their CiP around end of life care. There is a plan to extend to all IMT3 trainees rotating through our hospital trust. Ongoing evaluation is planned with review of resource allocation to assess the long-term sustainability and viability of the course.

\section{A COVID-19 END-OF-LIFE MULTI-PROFESSIONAL SIMULATION COURSE TO PREPARE STAFF FOR DIFFICULT CONVERSATIONS WITH PATIENTS AND RELATIVES DURING THE PANDEMIC}

Sabrina Vitello, Sally Middleton, Eloise Van Vuren, Jessica Wadsworth, James Horn. Epsom and St Helier University Hospitals NHS Trust

\subsection{6/spcare-2021-PCC.67}

Background Most healthcare professionals redeployed to COVID-19 critical care or cohort wards in our hospitals in the first wave expressed feelings of discomfort and inexperience speaking about death and dying especially with relatives over the phone. Although doctors were initially expected to take on this role, as the first wave progressed, more nurses and Allied Healthcare Professionals (AHPs) recognised the need to speak with relatives themselves as they answered phones or doctors were unavailable.

Methods We designed and delivered a half-day multi-professional simulation course involving two scenarios: an in-person ward conversation with the patient and their relative, and a phone conversation with a relative $\hat{a} €$ 'about clinical deterioration with COVID-19. One or two healthcare professionals were actively involved in each scenario while the rest of the group observed in another room. A whole group debrief followed. Interestingly, for the phone scenario, the cameras were set up so the observers could see both the healthcare professional's and relative's perspectives. Pre- and post-course surveys were completed by participants to evaluate the sessions.

Results 22 participants (doctors, nurses, AHPs and physician associates) attended across five sessions between 14th July and 11th November 2020. Pre and post-course scores showed increased confidence levels with discussing issues around death and dying including symptoms, psychosocial and spiritual needs with patients and relatives, especially over the phone. All staff recommended the course and felt scenarios were a good reflection of real-life situations. Qualitative data supported this and highlighted specific themes around preparation, phrases, non-verbal considerations for enhancing conversations. They also expressed the value of the multi-professional aspect of the course.

Conclusion There is need for more multi-professional courses encouraging junior doctors, nurses and AHPs to have conversations around death and dying. The half day duration of the course enabled both staff to be released from clinical duties to attend as participants and faculty.

\section{TIME CRITICAL TELEPHONE CONVERSATIONS IN THE EMERGENCY DEPARTMENT - A PILOT EDUCATIONAL PROJECT TO IMPROVE COMMUNICATION SKILLS OVER THE TELEPHONE WHEN BREAKING BAD NEWS TO RELATIVES}

Sarah Edwards, Lisa Keillor, Lorna Sandison, Abigail Millett, Ffion Davies. University Hospitals of Leicester, Leicester Royal Infirmary; Kingsmill Hospital, Sherwood Forest Hospitals NHS Trust, Nottinghamshire

\subsection{6/spcare-2021-PCC.68}

Background In many countries the COVID-19 pandemic has resulted in unwelcome news being delivered by staff over the telephone as hospital visiting by relatives has been restricted. This includes having to communicate patients have died or are critically unwell. We wanted to increase nursing and medical staff confidence and skills in communicating by telephone, in time critical situations to relatives in the emergency department.

Methods A blended learning package was created. This consisted of a bespoke 15-minute eLearning session and a 1-hour facilitated role-play session. Two simulated telephone calls to a professional actor, posing as the relative were undertaken. The calls simulated realistic time critical telephone conversations including obtaining crucial medical information, conveying news of an acutely unstable patient. A second call to the relative involved breaking the news that the patient had died. Following this the actor gave feedback to the caller focusing on the experience of a relative during these conversations with observers joining as a learning conversation. Group participants reflected on their learning during the session and in a follow up questionnaire. The actors were paid for by the department.

Results 61 staff received this session over the months May and June 2020. Key new elements specific to telephone conversations were explored. During the learning conversation, learners discovered important ideas for framing the 